\title{
Mass Spectrometry and the Amyloid Problem-How Far Can We Go in the Gas Phase?
}

\begin{abstract}
Alison E. Ashcroft
Astbury Centre for Structural Molecular Biology, Institute of Molecular and Cellular Biology, University of Leeds, Leeds, United Kingdom

A number of proteins are capable of converting from their soluble, monomeric form into highlyordered, insoluble aggregates known as amyloid fibrils. In vivo, these fibrils, which accumulate in organs and tissues, are associated with a wide range of amyloid diseases for which there are currently no therapeutic solutions. The molecular details of the pathway from native monomer through oligomeric intermediates to the final amyloid fibril remain a challenging enigma. Over the past few years, mass spectrometry has been applied to investigate the various stages of amyloid fibril formation, and this report summarizes the key steps achieved to date. (J Am Soc Mass Spectrom 2010, 21, 1087-1096) (C) 2010 American Society for Mass Spectrometry
\end{abstract}

A myloidosis has been puzzling scientists for some 150 years when the name amyloid, derived from amylum (Latin) and amylon (Greek), both meaning starch, was first proposed by Rudolph Virchow on account of the cellulose-like qualities of a brain tissue abnormality he had observed [1, 2]. In fact, amyloid deposits are composed of protein fibrils and in principle there is one type of protein in each case of amyloidosis [3]. There are $>20$ such (quite different) proteins that undergo amyloidosis in humans in vivo and which are associated with a variety of diseases, including Alzheimer's, Parkinson's, Huntington's, hemodialysis-related amyloidosis, and the spongiform encephalopathies (Table 1) [4]. Although these proteins have no apparent similarities in terms of amino acid sequence or tertiary structure, the fibrils they produce show a high degree of resemblance in their external morphology [5] and characteristics, which include low solubility and an unbranched structure with an extended cross- $\beta$ sheet central core that can be detected using X-ray diffraction [6], observation of birefringence with the dye Congo red [7], and chemical binding to Thioflavin T [8]. Moreover, it has been reported that amyloid formation is possible in vitro under chosen conditions for all peptide chains regardless of their sequence [9].

Although it is generally acknowledged that amyloidosis is a clinically and biochemically heterogeneous group of protein folding/misfolding disorders accompanied by self-aggregation, we have still much to learn about the amyloid fibril assembly pathways, precisely

Address reprint requests to Dr. A. E. Ashcroft, Astbury Centre for Structural Molecular Biology, Institute of Molecular and Cellular Biology, The University of Leeds, Leeds LS2 9JT, UK. E-mail: a.e.ashcroft@leeds.ac.uk which species are the culprits of disease, and how to prevent the debilitating diseases caused by this phenomenon. So what are the difficulties involved in understanding these polymerization reactions? The complexity of the situation is illustrated in Figure 1 [10]. First, the protein monomer may well be present in a co-populated mixture of folded, partially folded, and highly unfolded families of conformers, any one (or more) of which may be capable of initiating the assembly process [11]. Next, although early reports indicated that the amyloid fibrils were the primary pathogenic agent, recent evidence has suggested that smaller aggregates populated during fibril assembly are more likely to be responsible for pathogenesis [12-15]; for example, it has been suggested that $\mathrm{A} \beta$ oligomers could be the cause, rather than a product of, Alzheimer's disease [16]. This suggestion makes the characterization of all species present within the heterogeneous ensembles that exist during the aggregation process both necessary and highly important. Additional points of concern to the analyst are that fibril formation is stochastic, many of the noncovalently bound, macromolecular intermediate species are transient and present in barely discernible quantities, and also there is the possibility that some aggregation species may be deadend, rather than on-pathway, products. In fact, out of all the species to be considered within this complex reaction scheme, the amyloid fibril itself, despite its size and insolubility, is the easiest to identify [albeit not by mass spectrometry (MS)] due to its longevity and consistentlyordered structure.

Low-resolution details of intact amyloid fibrils, which can be generated by electron microscopy (EM) [17], cryo-EM [18], and atomic force microscopy (AFM) $[19,20]$, have shown that fully assembled amyloid 
Table 1. The more common amyloid diseases and their associated proteins

\begin{tabular}{|c|c|}
\hline Disease & Protein \\
\hline Alzheimer's & $\begin{array}{l}\mathrm{A} \beta, \text { from amyloid } \\
\quad \text { precursor protein (APP) }\end{array}$ \\
\hline Atherosclerosis & Apolipoprotein A1 \\
\hline Dialysis-related amyloidosis & $\beta_{2}$-Microglobulin $\left(\beta_{2} \mathrm{~m}\right)$ \\
\hline $\begin{array}{l}\text { Familial amyloid } \\
\text { polyneuropathy }\end{array}$ & Transthyretin \\
\hline $\begin{array}{l}\text { Familial non-neuropathic } \\
\text { amyloidosis }\end{array}$ & Lysozyme \\
\hline Huntington's & Huntingtin \\
\hline Injection-localized amyloidosis & Insulin \\
\hline Medullary thyroid carcinoma & Calcitonin \\
\hline Parkinson's & a-Synuclein \\
\hline Pituitary gland amyloidosis & Prolactin \\
\hline Primary systemic amyloidosis & $\begin{array}{l}\text { Immunoglobulin (lg) light } \\
\text { chain }\end{array}$ \\
\hline Rheumatoid arthritis & Serum amyloid $\mathrm{A}$ \\
\hline $\begin{array}{l}\text { Transmissible spongiform } \\
\text { encephalopathies }\end{array}$ & Prion \\
\hline Type II diabetes & $\begin{array}{l}\text { Islet amyloid polypeptide } \\
\text { (IAPP) }\end{array}$ \\
\hline
\end{tabular}

fibrils may contain different numbers of protofilaments, either intertwined into helices or bound side-to-side in a twisted ribbon structure [21, 22], in which the $\beta$-strands are arranged perpendicular to the fibril axis.
To date, the fully assembled fibrils have not been detected in their intact forms by MS, due to their size, intractability, and heterogeneity.

However, MS, and in particular electrospray ionization (ESI)-MS, comes into its own in the analysis of heterogeneous populations of soluble species of differing mass or mass-to-charge ratio $(\mathrm{m} / \mathrm{z})$ and, when coupled to ion mobility spectrometry (IMS), of differing collision cross-section also. Not only does MS provide the opportunity for such species to be identified-even as minor entities-within mixtures, it offers the means to monitor the appearance and disappearance of individual species during a reaction pathway, thus affording a distinct advantage over complementary biophysical techniques, including circular dichroism (CD), nuclear magnetic resonance (NMR), and Fourier transform infrared (FT-IR), which yield population-average data. Furthermore, the small amounts of sample required, the ability to maintain noncovalent interactions in the gas phase, and the speed of data acquisition render ESI-MS ideal for monitoring protein aggregation in real-time, directly from the reaction solution.

Despite this apparent panacea, there remain fundamental issues to consider: can MS, a gas-phase technique, reveal useful insights into solution phase amyloid fibril formation? How can we copy in vivo amyloid fibril formation in vitro, in particular under MS-compatible

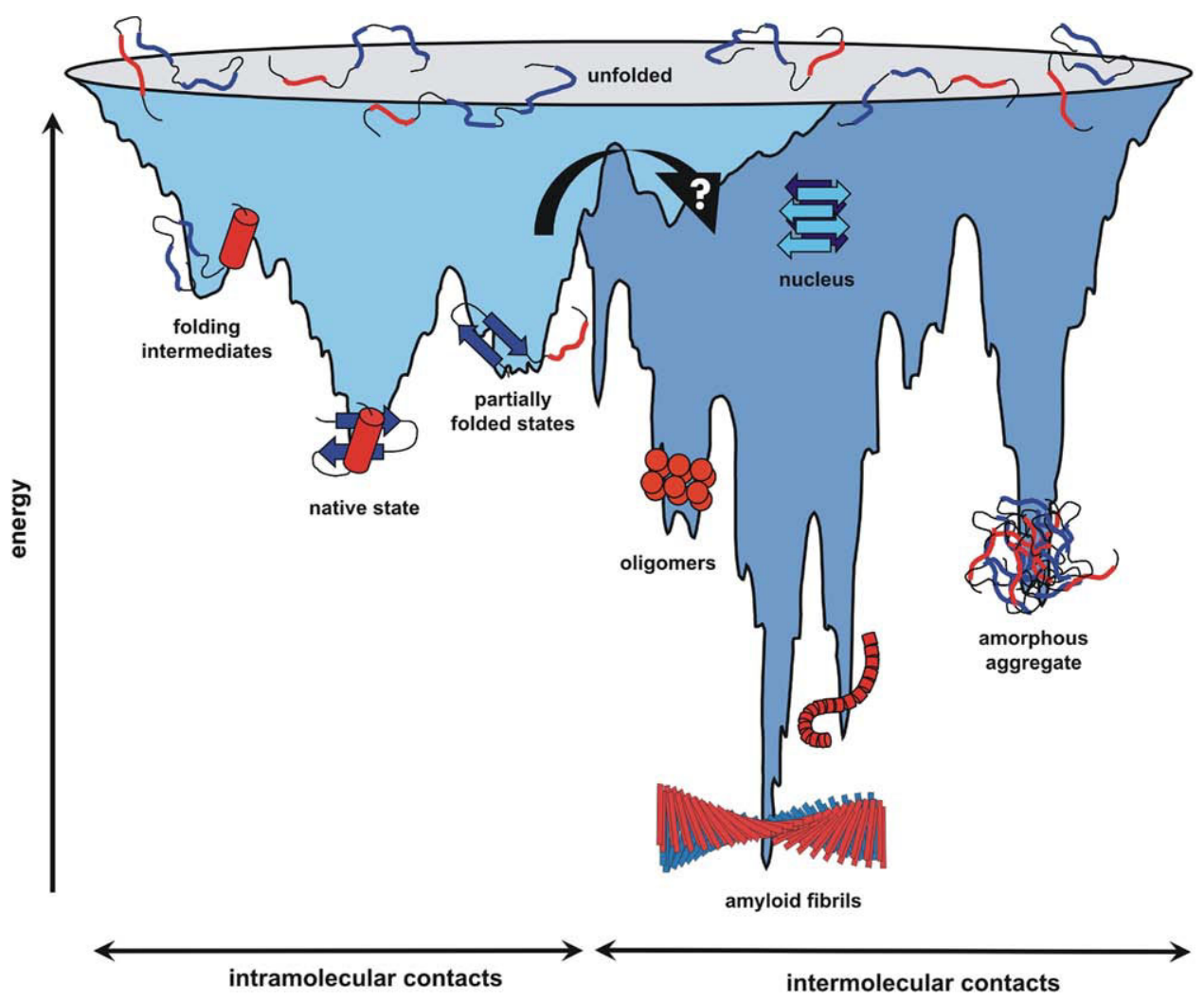

Figure 1. Taken from [10] (Figure 1). A schematic energy landscape for protein folding and aggregation. The surface shows the multitude of conformations 'funneling' towards the native state via intramolecular contact formation, or towards the formation of amyloid fibrils via intermolecular contacts. Reprinted by permission from FEBS Letters: FEBS Lett. 2005, 272, 5962-5970. 
conditions? As we are far from the stage where we can reproduce the complex environment of the cell perfectly in the laboratory, we must bear in mind that what we detect may not be relevant to in vivo amyloidosis and strive to obtain supporting information from a range of complementary biophysical and chemical techniques. The aim of this article is to assess how far we have advanced with our quest to decipher the intriguing questions posed by the amyloid problem using MS, in terms of protein folding and misfolding, oligomer formation during the assembly pathway, intact amyloid structure, and finally, ways in which we can influence assembly externally.

\section{Step-by-Step Amyloid Assembly with Mass Spectrometry}

\section{What Can We Learn About Protein Conformers?}

Early ESI-MS experiments illustrated that the $\mathrm{m} / \mathrm{z}$ charge state distribution of a protein depended on its folded state, with a more folded protein having a narrower charge state distribution with fewer charges on average than the same protein analyzed under alternative solution conditions which favor a less folded conformation [23]. To date, the relationship between ESI-MS charge state distributions (i.e., protein conformation in the gas phase) has correlated well with the conformational properties of proteins in solution, thus supporting the notion that protein structure in the solution phase can be retained after ionization and, for some time, into the gas phase [23, 24].

But what about a more realistic situation in which a protein could populate a number of conformational states simultaneously? With ESI-MS analysis, a number of overlapping charge state distributions may ensue, making it difficult to assign the number of conformational states present and the relative abundance of each one within an ensemble. As protein unfolding and subsequent misfolding are thought to be key initial steps in the amyloid-forming process, the identification of different conformeric entities within a co-populated ensemble of monomeric protein is of interest to amyloid researchers. This feat can be achieved by IMS, which has the ability to separate ions on account of their shape and charge and has been shown to be particularly successful when coupled to MS for separating a number of co-populated conformers of varying shape arising from a single protein [25-28]. This methodology has proved effective recently for separating and identifying co-populated conformers of the amyloidogenic protein $\beta_{2}$-microglobulin $\left(\beta_{2} \mathrm{~m}\right)$ [29]. $\beta_{2} \mathrm{~m}$, a 99-residue protein with a $\beta$-sheet structure associated with the disorder hemodialysis-related amyloidosis, is the light chain of the major histocompatibility complex class 1 (MHC-1). In vivo, monomeric $\beta_{2} \mathrm{~m}$ is shed continuously from the surface of cells displaying MHC-1 molecules and in healthy humans $\sim 95 \%$ of the free protein is removed through the kidney. Renal failure results in an increase in $\beta_{2}$ m concentration of $>25$-fold, leading to the deposition of amyloid plaques in the joints [30, 31]. $\beta_{2} \mathrm{~m}$ titration with concomitant detection and separation of the co-populated conformers at each $\mathrm{pH}$ was achieved [29] using the recently developed traveling wave IMS (TWIMS)-MS system [32, 33] (Figure 2). Simultaneous collision cross-section measurements of the protein conformers, achieved directly in conventional IMS-MS

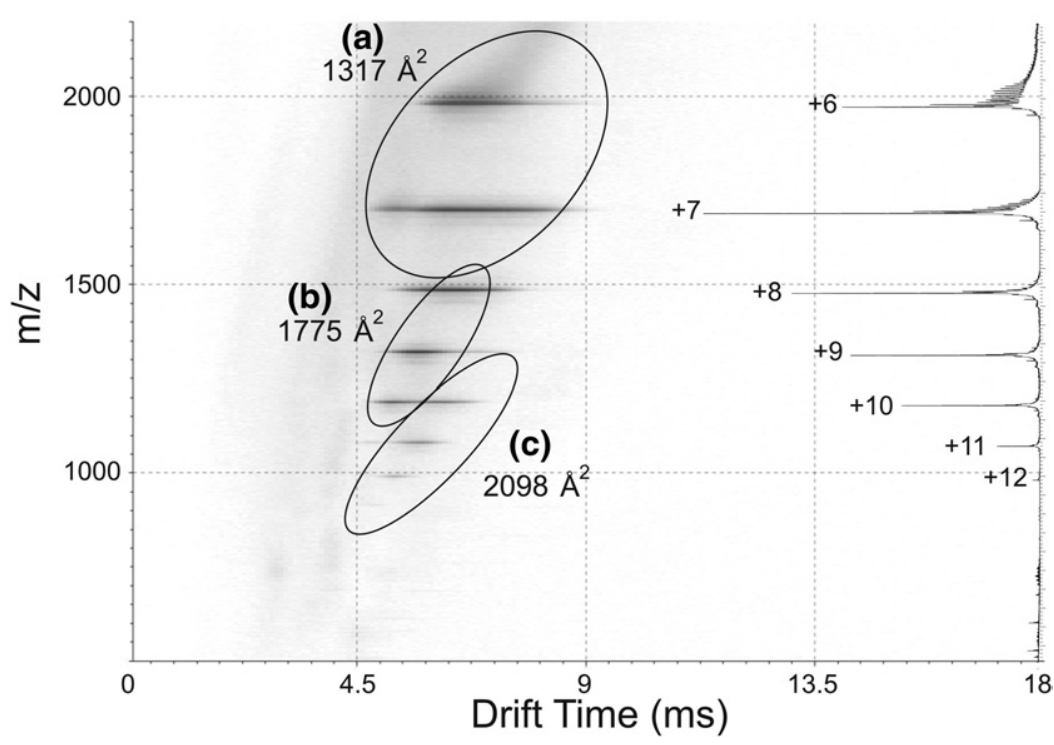

Figure 2. Taken from [29, 36] (Figure 7). ESI-TWIMS-MS data (drift time (mobility, ms) versus $\mathrm{m} / \mathrm{z}$ versus intensity) showing the detection of three co-populated families of conformers of the amyloidogenic protein $\beta_{2}$-microglobulin at $\mathrm{pH}$ 2.6. The folded (a), partially unfolded (b) and acid-unfolded (c) conformers have collision cross-sectional areas $(\Omega \mathrm{s})$ of $1317 \AA^{2}, 1775 \AA^{2}$, and $2098 \AA^{2}$, respectively. The inset on the right hand side shows the summed $\mathrm{m} / \mathrm{z}$ spectrum over the entire experiment. 
experiments [34, 35], or indirectly with TWIMS-MS [36-38] by calibration with a series of IMS-MS analyzed standards [39], were also made. This method has proven to be a useful and rapid method for assessing the possible correlation between protein stability and propensity for fibril formation, by comparing the wildtype protein with a series of amino acid variants [29, 36].

Consequently, ESI-IMS-MS has armed us with unique methodology that will measure the molecular mass of a protein, separate co-populated families of conformers within an ensemble, and provide information regarding the relative physical sizes of each of these conformeric groups in a single, rapid experiment, thus paving the way forwards for individual protein conformers to be monitored during fibril assembly, and their interactions with potential therapeutic inhibitors to be assessed.

\section{Are Oligomers Amyloid Intermediates or Dead-End Products and Can We Monitor Amyloid Pathways In Vitro?}

When analyzing noncovalently bound protein complexes, their tertiary and quaternary structure can be retained, at least to some extent, in the gas-phase during the ESI-MS analysis. Applications covering a wide range of complexes, often of very high molecular mass, now appear with regularity in the literature, including inter alia virus capsids [40], small heat shock proteins [41], myosin VI complexes [42], and hemocyanins [43]. Thus, the study of oligomers arising from amyloidogenic proteins, which is of high importance not only in determining the amyloid pathway but also because recent reports have indicated that oligomers may play a key role in amyloid-related disease, is achievable.

Furthermore, the recent upsurge in interest in IMS-MS has made a distinct impact on the characterization of the 3D structures of noncovalently bound complexes. In the case of amyloid-related oligomers, information on the shape of each oligomeric species has been achieved, either directly by conventional IMS-MS [44] or indirectly by TWIMS-MS [45]. By comparing the measured cross-sectional areas with values calculated from 3D coordinates using modeling programs, potential structural arrangements of subunits can be considered for these complex biomolecules. The freely-available, web-based Mobcal suite of modeling programs is frequently used for this purpose. Mobcal consists of three different programs: the projection approximation method originally from the Bowers' group [46] and the trajectory method and exact hard sphere scattering calculations from Jarrold [47, 48]. More recent variations on the projection approximation method have emerged [36, 49, 50].

Of course, there is always the ubiquitous question of whether the oligomers present, both in solution and in the gas phase, are true on-pathway amyloid intermediates or whether they are off-pathway aggregation spe- cies. This is an important issue that has been reviewed by others, including discussions of a range of structural, kinetic, and thermodynamic experimental evidence $[22,51-53]$ and of the diversity of kinetic pathways in amyloid formation [54].

Additionally, a number of concerns arise specifically when considering the detection of protein aggregates by ESI-MS. Is it possible to identify a multitude of oligomers arising from a single protein unambiguously, when they are likely to give rise to ions of the same $\mathrm{m} / \mathrm{z}$ ratios? Are the gas-phase oligomers we detect representative of the oligomers present in solution or could they be artefacts of the ionization technique? Are some species being suppressed during the analysis of these heterogeneous mixtures? How do we know that fibril formation in vivo can be reproduced in vitro, often in buffers and solvents chosen for their MS-compatibility rather than any physiologic reasons? The greater the number of manuscripts that appear describing noncovalent protein complex analysis by MS, the more the general scientific public accepts that MS gas-phase studies can generate meaningful information on biomacromolecular systems. However, in practice it is sensible to corroborate results with data from complementary biophysical techniques, whenever possible, to avoid overenthusiastic conclusions being made. The following case studies cover examples where these issues have been successfully addressed.

An early paper reporting the ESI-MS detection of oligomers in protein self-assembly systems focused on insulin, a 51-amino acid residue protein, which forms well-defined oligomers in its native state yet can be induced to aggregate into amyloid fibrils under nonphysiologic conditions [55]. In vivo, insulin is stored in the pancreas as a $\mathrm{Zn}^{2+}$-containing hexamer composed of three equivalent dimers, and it is this form that is used in the treatment of type 1 diabetes. Following administration, to achieve more efficient absorption into the blood stream, the hexamers are broken down into monomers and dimers, which are prone to fibrillar self-assembly, thus limiting the therapeutic value of this approach. ESI-MS analysis of insulin in the presence of $\mathrm{Zn}^{2+}$ led to the detection of monomers, dimers, tetramers, and hexamers (the latter with two to four $\mathrm{Zn}^{2+}$ ions bound) [55]. The absence of odd-numbered oligomers indicated that such species were not stable entities in solution under these conditions and provided good evidence that the oligomers detected had not been generated artefactually during the ESI-MS process. At lower $\mathrm{pH}$, conditions under which insulin is known to aggregate in solution, populations of higher order oligomers up to and including the dodecamer were detected. Increasing the temperature of the solution caused the population of these oligomers to decline rapidly, suggesting that their demise was connected with the formation of higher order aggregates and fibrils, the latter confirmed by EM.

A contemporary report was published on islet amyloid polypeptide (IAPP), a 37-residue C-terminally ami- 
dated peptide hormone, which is co-secreted with insulin in the pancreas and, in its fibrillar form, is the main component of diabetes-related amyloid plaques [56]. ESI-MS was used to follow human IAPP amyloid formation by monitoring monomer consumption, whilst IAPP from rat (of similar ESI-MS sensitivity to human IAPP) was employed as a non-fibrillogenic control. Using this methodology, precursor consumption in seeded reactions was found to follow first-order kinetics, although the study did not identify any oligomeric species [56].

ESI-MS studies on the presence of $\beta_{2}$-microglobulin oligomers in amyloid fibril assembly in vitro were complemented by analytical ultracentrifugation (AUC) data to confirm the presence of oligomers in solution, and formation of the final fibrils was verified by a Thioflavin-T fluorescence test [57]. The ESI-MS data indicated that whereas the worm-like fibrils, which form with nucleation-independent kinetics at $\mathrm{pH}$ 3.6, assemble by a mechanism consistent with sequential monomer addition with species ranging from monomer to 13-mer (inclusive) being detected en masse as transient assembly intermediates, in contrast only monomers, dimers, trimers, and tetramers were observed during nucleated fibril growth at $\mathrm{pH} 2.5$, leading to the formation of long, straight fibrils very similar in appearance to those found ex vivo. These heterogeneous ensembles led to complicated spectra from which it was difficult to assess the role in the assembly pathway of any particular oligomer [57]. More recent ESI-IMS-MS studies have succeeded in separating the oligomers during the analysis, thus making possible the interrogation of individual oligomers [45].

Much research is directed at the widespread Alzheimer's disease in which the associated fibrils comprise proteolytic fragments $(\mathrm{A} \beta)$ of some 39 to 43 residues derived from the 753-residue $\beta$-amyloid precursor protein (APP) [58]. An early publication in this area focused on a model peptide corresponding to A $\beta 10-30$, which contains the core region involved in $A \beta$ fibrillar aggregation. Detection of A $\beta 10-30$ oligomers by ESI-MS in $10 \mathrm{mM}$ ammonium acetate solution led to the identification of monomer, dimer, trimer, tetramer, pentamer, and hexamer [59]. From the highest amplitude charge states observed for each oligomer, it was found that the charge per monomeric subunit within these oligomers decreased from monomer $(+3)$ to pentamer $(\sim+1.5)$, but then increased again for hexamer $(+1.8)$. Thus, from monomer to pentamer a gradual build-up of structural compactness was observed, whilst for the hexamer, the charge per monomeric subunit value reverted almost to that of the dimer $(+2)$, indicating a gross change in structural organization. Interestingly, regardless of the peptide's concentration, the sum of the oligomeric peaks never exceeded $10 \%$ of the total response. To gain further insight into the structural properties of these oligomers, isotope exchange studies, in conjunction with ESI-MS, were performed. Hydrogen deuterium exchange (HDX) indicated no difference in the rate of exchange for monomers, dimers, and trimers at neutral $\mathrm{pH}$, suggesting either that there is a lack of defined, stable structure in these oligomers or that there is a fast exchange between oligomer subunits and the pool of monomers. To measure the exchange rate between oligomers and the pool of monomers directly, ${ }^{15} \mathrm{~N}$-labeled $\mathrm{A} \beta 10-30$ was mixed with unlabeled peptide and analyzed. Dimers and trimers both gave the expected ratio of unlabeled, mixed-labeled, and labeled subunits (1:2:1 and 1:3:3:1, respectively), implying that full subunit exchange had been achieved in a subsecond time scale, and thus confirming that the lack of hydrogen shielding observed in the HDX experiment results from fast monomer subunit exchange and does not implicate a lack of definite structure in these oligomers.

A recent publication reported the elegant separation and characterization of a number of co-populated $\mathrm{A} \beta$ oligomers using conventional IMS-MS [44, 60-62]. This study indicated differences in the oligomers formed from $A \beta 1-42$ compared with those formed from $A \beta 1-$ 40 , two species that adopt similar random coil structures in solution, although $\mathrm{A} \beta 1-42$ forms amyloid fibrils significantly faster than $A \beta 1-40[44,60]$. In vitro, $A \beta 1-42$ showed evidence for dimer, tetramer, hexamer, and dodecamer oligomers, whilst only the dimer and tetramer were detected in the case of $A \beta 1-40$, thus offering plausible insights into why their contribution to amyloidosis is quite different (Figure 3). Collision crosssections were measured directly from the IMS-MS experiment and modeling undertaken to probe potential structures of the species measured. The modeling in this case assumed each monomer to have a spherical shape and from this the average cross-sectional area was calculated using the projection approximation method [46]. Following on from this, an overlap of some $10 \%$ of monomer spheres was found to reproduce the experimental cross-sectional area of the dimer, and this adjustment was used to model the higher-order oligomers in this study. In the case of the $A \beta 1-42$ hexamer, a modeled ring-like arrangement of monomers had the closest calculated collision cross-section to that measured, compared with both straight chain and spherical models. The A $\beta 1-42$ tetramer had a collision crosssection which was consistent with a model of an angled segment of the ring-like hexamer, with quite an open structure affording sufficient space for subsequent dimer addition to complete the ring and form the hexamer. In contrast, the $\mathrm{A} \beta 1-40$ tetramer was found to have a smaller collision cross-section indicating a more closed structure, which would not be disposed to the addition of either a monomer or a dimer and hence explaining why there was no evidence of the hexamer or any higher order species for A $\beta 1-40$. From these [44] and earlier [60] ESI-IMS-MS oligomer data, a global scheme of amyloid- $\beta$ oligomerization was proposed in which the $A \beta 1-42$ tetramer added a dimer to produce the ring-shaped hexamer, which subsequently dimerized to produce a two-ring, stacked dodecamer (Figure 3). Following this, it was suggested that the dodecamer 


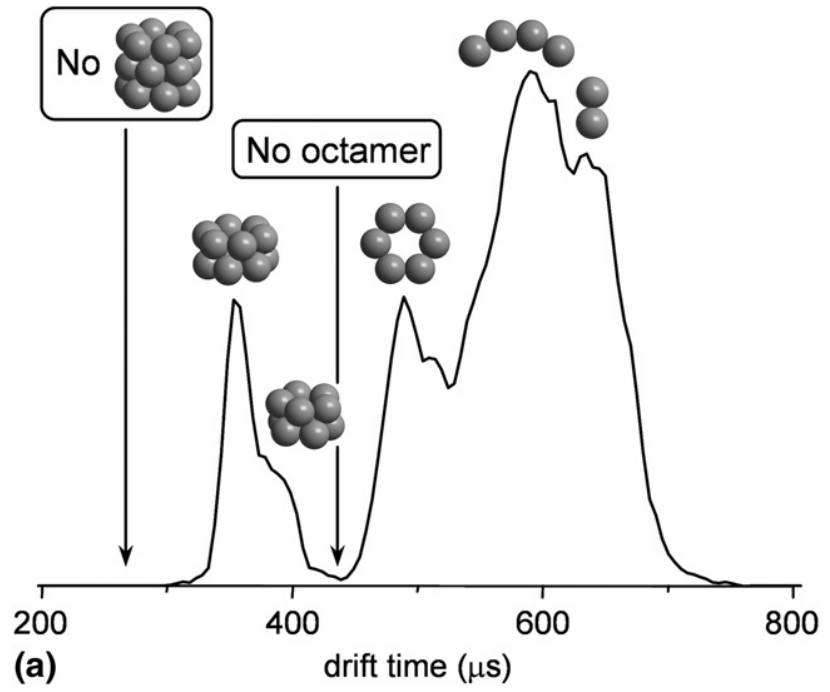

(b)

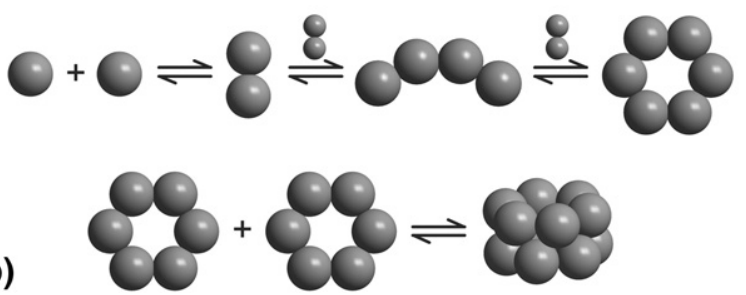

Figure 3. Taken from [44] (Figure 3). (a) The arrival time distributions (drift time $(\mu \mathrm{s})$ versus relative intensity) of $\mathrm{A} \beta 1-42$ oligomers (represented by the $z / n=-5 / 2$ charge state) observed by conventional IMS-MS with structural designations in place; (b) a plausible mechanism for forming the oligomer distribution shown in (a). Reprinted by permission from Macmillan Publishers Ltd: Nat. Chem. 2009, 1, 326-331.

underwent rearrangement, added more monomers and formed $\beta$-sheet fibrils. In the case of $\mathrm{A} \beta 1-40$, the more compact tetramer was proposed to undergo very slow fibril formation, although this was not observed during the time-scale of these experiments. This study indicates the amount of detail that can be obtained simultaneously on a number of oligomeric species within a heterogeneous ensemble.

Transmissible spongiform encephalopathies (TSEs), often referred to as prion diseases, represent a group of fatal neurodegenerative disorders associated with the accumulation of amyloid fibrils that arise in mammalian species and include bovine spongiform encephalopathy in cattle, scrapie in sheep, and CreutzfeldtJakob disease in humans. These fibrillar aggregates result from the conversion of the normal cellular prion protein $(\mathrm{PrP})$ to an abnormal conformer, $\mathrm{PrP}^{\mathrm{Sc}}$, and although $\mathrm{PrP}$ and $\mathrm{PrP}^{\mathrm{Sc}}$ appear to share the same covalent structure, they differ in biophysical properties [63]. In an in vitro study of mouse and Syrian hamster recombinant PrP proteins, both formed aggregates but each with a distinct morphology: the mouse fibrils were formed by twisted protofibrils whereas the hamster fibrils had a diameter some $\sim 50 \%$ less. This study demonstrated also that these $\sim 16 \mathrm{kDa}$ proteins can adopt the $\alpha$-helical native isoform, two non-native $\beta$-sheet-rich isoforms, a $\beta$-oligomer, and an amyloid fibril. The preferences for forming either a $\beta$-oligomer or amyloid can be dictated by experimental conditions, with acidic conditions favoring the former and neutral conditions the latter. ESI-MS analysis of the $\beta$-oligomer indicated this species to be predominantly octameric $(\sim 129 \mathrm{kDa})$ but its biological significance has not yet been determined [63]. A more recent study compared the monomeric and oligomeric structures of normal PrP106-126 (the protein domain thought to be involved in amyloid formation) and two non-aggregating forms of the peptide, an oxidized form in which both methionine residues are oxidized to methionine sulphoxide and a control peptide consisting of the same amino acids as PrP106-126 in a scrambled sequence. Oligomers were detected for the wild-type peptide only and molecular dynamics simulations were carried out to explore the relationship between sequence and structure of the three peptides [64].

\section{What Can We Learn About Amyloid Fibrils?}

Despite details concerning the oligomeric states detected en route to fibril formation beginning to emerge, the question of how the polypeptide chains are wound into the generic cross- $\beta$ structure of amyloid fibrils still remains elusive. Although the MS analysis of intact fibrils has not yet been reported, proteolysis followed by MS(/MS) characterization of the cleaved peptide fragments has been used to generate details of the structural organization of the fibrillar structure. In general, proteolysis of the intact fibril (either directly or after HDX), followed by defibrillization and mass spectrometric analysis of the protein and resulting peptides, has provided significant insights into the structural characteristics of amyloid fibrils [65].

HDX offers insights into the extent of stable protein structure and is therefore important for studying amyloid due to the characteristic, highly stable, $\beta$-sheet fibril architecture. The experimental procedure generally involves the independent exposure of both the protein monomer and the amyloid fibrils to deuterated solvent for a specific time, after which the extent of deuteration in each is measured by MS and compared. In the case of the protein monomer, the deuteration level can be measured on either the intact protein [66] or, to scrutinize specific amino acid residues, on peptides arising from protein proteolysis [65, 67]. The deuterated fibrils first need to be dissolved in dimethylsulphoxide to regenerate monomer before being subjected to proteolysis and analysis [65]. This approach has been applied to a number of amyloid systems. In one such case, two amyloidogenic variants of lysozyme were compared with wild-type protein and were found to have unfolded regions in the $\beta$-domain and adjacent C-helix [67]. Structural differences between A $\beta 1-40$ protofibrils and fibrils have also been reported [68]: although the Cand N-terminal fragments in both the fibrils and proto- 
fibrils were found to be highly exposed to HDX, the internal peptide fragment consisting of residues 20-34 was found to be highly protected in fibrils, but not so in protofibrils, suggesting that although the $\beta$-sheet elements in the final fibrils may be present to some extent in the protofibrils, the transition from protofibril to fibril does involve significant ordering of this central region.

The recombinant, 289-residue HET-s prion protein analogue aggregates in vitro to produce infectious amyloid fibrils. HDX of these fibrils followed by pepsin proteolysis and matrix-assisted laser desorption ionization (MALDI)-MS analysis indicated that the prionforming domain (corresponding to C-terminal residues 218-289) is highly protected in amyloid [69-71]. The HDX time-course also indicated that deuterium incorporation in the C-terminal region of the protein was drastically reduced in amyloid compared with the monomer [69].

The HDX properties of insulin under amyloid fibrilforming conditions have been explored with the results indicating that the protected regions of the protein lie in the A13-A19, A2-A8, and B chain helices, consistent with insulin being in a predominantly helical conformation under these conditions [72].

SH3 domains are small modules of $\sim 50$ amino acid residues found in many proteins involved in intracellular signal transduction and have a characteristic $\beta$-barrel fold consisting of five or six $\beta$-strands arranged as two antiparallel $\beta$-sheets. The amyloid-forming properties of the SH3 domain of bovine phosphatidylinositol-3-kinase (PI3-SH3), an 86-residue protein, have been interrogated in vitro $[12,73]$. Under acidic $\mathrm{pH}$ conditions, P13-SH3 adopts a compact denatured state which slowly forms a gel that consists of typical amyloid fibrils [74]. During an HDX-ESI-MS study to probe the nature of this amyloid structure, the fibrils were exposed to deuterated buffer for varying lengths of time and then solubilized into monomers using dimethylsulphoxide before analysis [73]. ESI-MS, with its ability to detect coexisting populations of molecules with different degrees of exchange, showed two well-resolved peaks on HDX exposure, indicating that two distinct isotopically labeled populations are present within the amyloid fibrils: one representing an exchange of $\sim 50 \%$ of the labile hydrogen atoms, the other almost complete exchange. The explanation proposed suggested that an ensemble of molecules within the fibrils is in dynamic equilibrium with a pool of soluble protein monomers (Figure 4), i.e., protein monomers dissociate from the fibrils, undergo HDX when in solution, and are then re-incorporated into the fibrils. Thus, two peaks are observed: one that represents the population of molecules not yet dissociated from fibrils, while the other represents the population that has dissociated, exchanged, and been re-incorporated into fibrils. Supporting this molecular recycling model, it was found that there is always a certain amount of soluble protein monomer in the fibril-containing mixture. This impor-

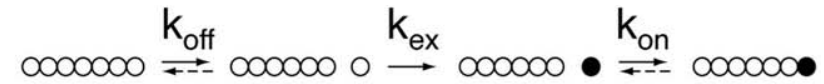

(a)

Initial time

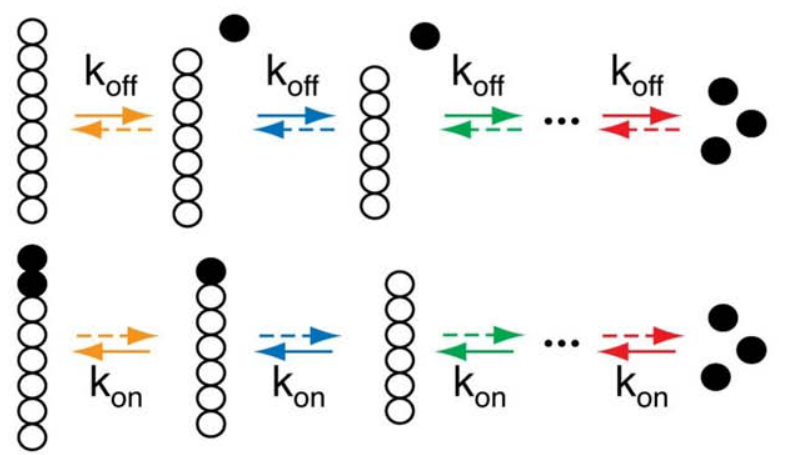

\section{Later time}

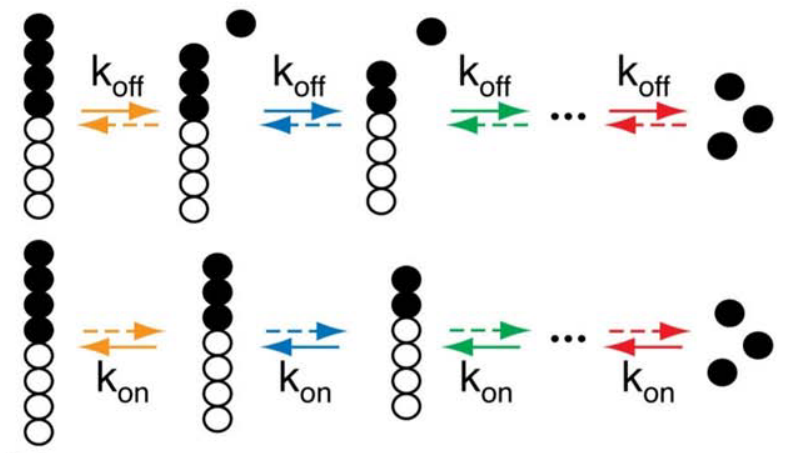

(b)

Figure 4. Taken from [73] (Figure 4). Schematic representation of the recycling process involving a single amyloid fibril. (a) A protonated protein molecule (open circle) dissociates from the fibril. Once in solution, HDX takes place rapidly and the molecule is subsequently reincorporated in a fully deuterated state (filled circle); (b) schematic representation of the recycling mechanism for a distribution of amyloid fibrils at different times of exchange; at a given time point the dissociation of a molecule from a fibril (upper) is counteracted by the re-association of that molecule in another fibril (lower). Reprinted by permission from Macmillan Publishers Ltd.: Nature 2005, 436, 554-558.

tant insight into the dynamic nature of the amyloid fibrils has implications for the design of therapeutics.

$\beta_{2} \mathrm{~m}$ amyloid fibrils have been the subject of several investigative structural studies using limited proteolysis followed by MS(/MS) analysis [75-78]. Comparisons of the surface topology of fibrils arising from the intact protein with that of fibrils formed from a truncated species lacking the first six amino acid residues $(\Delta \mathrm{N} 6-$ $\beta_{2} \mathrm{~m}$; which is also found in ex vivo fibrils) have been made [75; 77]. Both sets of fibrils were found to have a fully protected central region, comprising residues 20 87, and exposed C- and N-terminals. Limited proteolysis of $\beta_{2} \mathrm{~m}$ amyloid fibrils followed by ESI-MS(/MS) characterization of the resulting peptides illustrated clear differences in the architecture of fibrils formed from partially unfolded $(\mathrm{pH} 3.6)$ and acid-unfolded $(\mathrm{pH}$ 
2.5) protein monomer [76]. Despite having very different morphologic properties, both types of fibril show consistency with the common cross- $\beta$ configuration of amyloid. This study found that the long, straight, $\mathrm{pH}$ 2.5 fibrils (similar to ex vivo fibrils) were highly resistant to proteolysis and that $\sim 90 \%$ of the polypeptide chain was involved in the fibril core. Compared with this, the shorter, worm-like $\mathrm{pH} 3.6$ fibrils were more susceptible to proteolysis, and their protected core was reduced to $\sim 30 \%$ of the protein. Particular differences arose in strands B and F, close to the Cys25-Cys80 disulphide bond, which were protected in the long, straight fibrillar structures but less so in the worm-like fibrils, and also in the C-terminal region, which was involved in the protected core in the $\mathrm{pH} 2.5$ fibrils but not in the $\mathrm{pH} 3.6$ fibrils. The N-terminal 10 residues were highly susceptible to proteolysis in both types of fibrils.

\section{Can We Prevent Amyloid Formation?}

In addition to satisfying scientific curiosity, one of the key driving forces behind amyloid research must be the long-term goal of therapeutic design to prevent these fatal, and currently, unstoppable, diseases. One approach may be to inhibit the formation of fibrils, but the reported oligomer toxicity $[59,79,80]$ indicates that therapeutic strategies aimed at the destabilization of fibrils may prove counter-productive as this could lead to an increase in the level of oligomers.

A review some years ago highlighted a range of different compound classes including dyes (e.g., Congo red), rifamycin antibiotics, anthracycline derivatives, acridinones, benzofurans, and peptides, which had been found to inhibit the formation of $\mathrm{A} \beta$ fibrils. This behavior had been detected using biophysical techniques, such as light scattering, dye binding, EM, and AFM [79]—but not mass spectrometry! However, a high-throughput, sensitive, MS-based screening assay has been reported for potential $A \beta 1-40$ protein aggregation inhibitors: the procedure involves incubation of A $\beta 1-40$ with test compounds, followed by separation of the residual monomer from any aggregates, and then quantification of the monomer using ESI-MS (Figure 5) [81]. The greater the concentration of residual monomer indicates a greater degree of inhibition of aggregation, and the order of inhibition for the compounds screened in this study was: daunomycin $>3$-indolepropionic acid $>$ melatonin $>$ methysticin.

$\alpha$-Synuclein, a 140-amino acid residue presynaptic brain protein, is a major fibrillar component of Lewy bodies and hence associated with the loss of brain cells resulting in Parkinson's disease. The inhibitory effect in vivo of the antibiotic rifampicin on $\alpha$-synuclein aggregation, and also its ability to disassociate preformed fibrils, has been reported [80]. The data suggest that such inhibition is due to the stabilization of the protein monomer and soluble, low order oligomers. ESI-MS was used to examine $\alpha$-synuclein after incubation in the presence and absence of rifampicin; as no change to the protein was detected, covalent modification was ruled out [80].

A recent study whereby the effect of a single-domain fragment of a camelid heavy-chain antibody, specific for the active site of human lysozyme, on two amyloidogenic variants of this protein, was investigated [82].

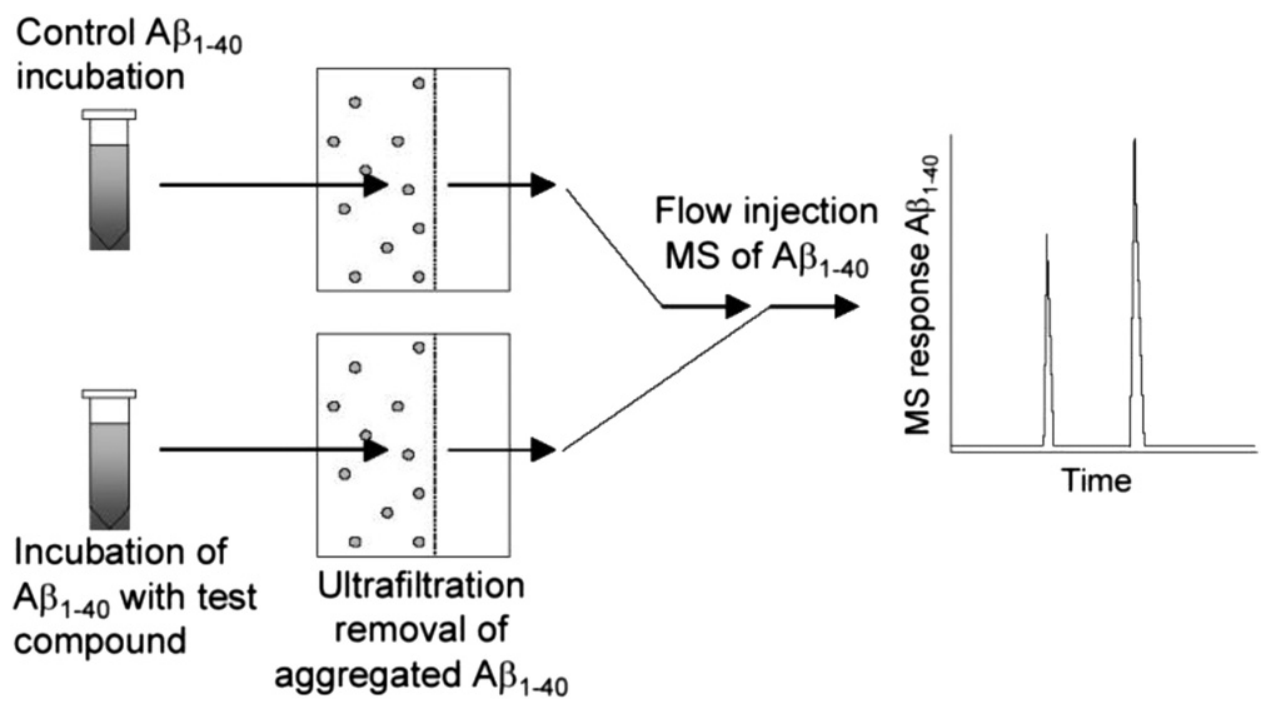

Figure 5. Taken from [81] (Figure 2). The ultrafiltration ESI-MS screening assay to test for ligand inhibition of $\mathrm{A} \beta$ aggregation. After incubation of $\mathrm{A} \beta 1-40$ with a test compound, any aggregated protein is removed by ultrafiltration and the residual protein monomer is quantified by comparison with the amount remaining in an untreated $A \beta 1-40$ solution. Compounds that prevent $A \beta 1-40$ aggregation produce solutions with the highest concentration of $A \beta 1-40$ monomer. Reprinted with permission from Analytical Chemistry: Anal. Chem. 2005, 77, 7012-7015. Copyright 2005, American Chemical Society. 
HDX-ESI-MS analysis indicated that antibody binding inhibited unfolding of the protein monomer. A more stable, disulphide-bridged version of the antibody was found to inhibit lysozyme fibril formation, suggesting that molecular targeting of enzyme-active sites, and consequently of protein binding sites, is an effective strategy for inhibiting self-assembly.

\section{Future Directions}

How far have we come with the amyloid problem in the gas phase? Co-populated protein conformers have been detected and separated, protein monomer consumption during fibril assembly has been assessed, the appearance and decline of oligomers - which have been identified by their mass and physical shape-have been measured during self-aggregation, and approaches to amyloid inhibition are now being reported. However, there is no respite as there remains much to achieve, especially in terms of defining the pathway from oligomers to the final fibrillar structures, and in identifying therapeutic remedies.

\section{Acknowledgments}

The author thanks her colleague and collaborator Professor Sheena E. Radford for introducing her to the amyloid problem and also their co-supervised amyloid-mass spectrometry post-doctoral fellows and graduate students past and present over the years. Lucy A. Woods is thanked for provision of the graphical abstract. Financial support for the amyloid-mass spectrometry research at the University of Leeds is gratefully acknowledged from the Biotechnology and Biological Sciences Research Council, the Wellcome Trust, the University of Leeds, the British Mass Spectrometry Society (travel grants), and Waters/Micromass UK Ltd. (Industrial CASE Ph.D. studentships).

\section{References}

1. Sipe, J. D.; Cohen, A. S. Review: History of the Amyloid Fibril. J. Struct. Biol. 2000, 130, 88-98.

2. Puchtler, H.; Sweat, F. A Review of Early Concepts of Amyloid in Context with Contemporary Chemical Literature from 1839 to 1859. J. Histochem. Cytochem. 1966, 14, 123-134.

3. Westermark, P.; Benson, M. D.; Buxbaum, J. N.; Cohen, A. S.; Frangione, B.; Ikeda, S. I.; Masters, C. L.; Merlini, G.; Saraiva, M. J.; Sipe, J. D. A Primer of Amyloid Nomenclature. Amyloid 2007, 14, 179-183.

4. Sacchettini, J. C.; Kelly, J. W. Therapeutic Strategies for Human Amyloid Diseases. Nat. Rev. Drug Discov. 2002, 1, 267-275.

5. Stefani, M.; Dobson, C. M. Protein Aggregation and Aggregate Toxicity: New Insights Into Protein Folding, Misfolding Diseases and Biological Evolution. J. Mol. Med. 2003, 81, 678-699.

6. Sunde, M.; Blake, C. The Structure of Amyloid Fibrils by Electron Microscopy and X-Ray Diffraction. Adv. Protein Chem. 1997, 50, 123-159.

7. Puchtler, H.; Sweat, F.; Levine, M. On the Binding of Congo Red by Amyloid. J. Histochem. Cytochem. 1962, 10, 355-364.

8. Le Vine, H. R. Quantification of $\beta$-Sheet Amyloid Fibril Structures with Thioflavin T. Methods Enzymol. 1999, 309, 274-284.

9. Dobson, C. M. Protein Folding and Misfolding. Nature 2003, 426, $884-890$.

10. Jahn, T. R.; Radford, S. E. The Yin and Yang of Protein Folding. FEBS Lett. 2005, 272, 5962-5970.

11. Platt, G. W.; Radford, S. E. Glimpses of the Molecular Mechanisms of B2-Microglobulin Fibril Formation In Vitro: Aggregation on a Complex Energy Landscape. FEBS Lett. 2009, 583, 2623-2629.

12. Carulla, N.; Zhou, M.; Arimon, M.; Gairdi, M.; Giralt, E.; Robinson, C. V.; Dobson, C. M. Experimental Characterization of Disordered and Ordered Aggregates Populated During the Process of Amyloid Fibril Formation. Proc. Natl. Acad. Sci. U.S.A. 2009, 106, 7828-7833.
13. Haass, C.; Selkoe, D. J. Soluble Protein Oligomers in Neurodegeneration: Lessons from the Alzheimer's Amyloid $\beta$-Peptide. Nat. Rev. Mol. Cell. Biol. 2007, 8, 101-112.

14. Kirkitadze, M. D.; Bitan, G.; Teplow, D. B. Paradigm Shifts in Alzheimer's Disease and Other Neurodegenerative Disorders: The Emerging Role of Oligomeric Assemblies. J. Neurosci. Res. 2002, 69, 567-577.

15. Bucciantini, M.; Giannoni, E.; Chiti, F.; Baroni, F.; Formigli, L.; Zurdo, J.; Taddei, N.; Ramponi, G.; Dobson, C. M.; Stefani, M. Inherent Toxicity of Aggregates Implies a Common Mechanism for Protein Misfolding Diseases. Nature 2002, 416, 507-511.

16. Finder, V. H.; Glockshuber, R. Amyloid- $\beta$ Aggregation. Neurodegener. Dis. 2007, 4, 13-27.

17. Kudva, Y. C.; Mueske, C.; Butler, P. C.; Eberhardt, N. L. A Novel Assay In Vitro of Human Islet Amyloid Polypeptide Amyloidogenesis and Effects of Insulin Secretory Vesicle Peptides on Amyloid Formation. Biochem. J. 1998, 331, 809-813.

18. White, H. E.; Hodgkinson, J. L.; Jahn, T. R.; Cohen-Krausz, S.; Gosal, W. S.; Muller, S.; Orlova, E. V.; Radford, S. E.; Saibil, H. R. Globular Tetramers of $\beta(2)$-Microglobulin Assemble into Elaborate Amyloid Fibrils. J. Mol. Biol. 2009, 389, 48-57.

19. Kheterpal, I.; Wetzel, R.; Cook, K. D. Enhanced Correction Methods for Hydrogen-Exchange Mass Spectrometric Studies of Amyloid Fibrils. Prot. Sci. 2003, 12, 635-643.

20. Gosal, W. S.; Myers, S. L.; Radford, S. E.; Thomson, N. H. Amyloid Under the Atomic Force Microscope. Protein Pept. Lett. 2006, 13, 261-270.

21. Kad, N. M.; Myers, S. L.; Smith, D. P.; Smith, D. A.; Radford, S. E.; Thomson, N. H. Hierarchical Assembly of $\beta 2$-Microglobulin Amyloid in Vitro Revealed by Atomic Force Microscopy. J. Mol. Biol. 2003, 330, 785-797.

22. Jahn, T. R.; Radford, S. E. Folding Versus Aggregation: Polypeptide Conformations on Competing Pathways. Arch. Biochem. Biophys. 2008, 469, 100-117.

23. Mirza, U. A.; Cohen, S. L.; Chait, B. T. Heat-Induced Conformational Changes in Proteins Studied by Electrospray Ionization Mass Spectrometry. Anal. Chem. 1993, 65, 1-6.

24. Kaltashov, I. A.; Eyles, S. J. Studies of Biomolecular Conformations and Conformational Dynamics by Mass Spectrometry. Mass Spectrom. Rev. 2002, 21, 37-71.

25. Clemmer, D. E.; Hudgins, R. R.; Jarrold, M. F. Naked Protein Conformations: Cytochrome $c$ in the Gas Phase. J. Am. Chem. Soc. 1995, 117, 10141-10142.

26. Clemmer, D. E.; Jarrold, M. F. Ion Mobility Measurements and Their Applications to Clusters and Biomolecules. J. Mass Spectrom. 1997, 32, 577-592.

27. Henderson, S. C.; Valentine, S. J.; Counterman, A. E.; Clemmer, D. E. ESI/Ion Trap/Ion Mobility/Time-of-Flight Mass Spectrometry for Rapid and Sensitive Analysis of Biomolecular Mixtures. Anal. Chem. 1999, 71, 291-301.

28. Kanu, A. B.; Dwivedi, P.; Tam, M.; Matz, L.; Hill, H. H. Ion MobilityMass Spectrometry. J. Mass Spectrom. 2008, 43, 1-22.

29. Smith, D. P.; Giles, K.; Bateman, R. H.; Radford, S. E.; Ashcroft, A. E. Monitoring Co-Populated Conformational States During Protein Folding Events Using Electrospray Ionization-Ion Mobility SpectrometryMass Spectrometry. J. Am. Soc. Mass Spectrom. 2007, 18, 2180-2190.

30. Floege, J.; Ketteler, M. $\beta$-2-Microglobulin Derived Amyloidosis: An Update. Kidney Int. 2001, 78(Suppl.),S164-S171.

31. Gejyo, F.; Yamada, S.; Odani, Y.; Nakagawa, M.; Arakawa, T.; Kunitomo, H.; Kataoka, M.; Suzuki, Y.; Hirasawa, T.; Shirama, T. A New Form of Amyloid Protein Associated with Chromic Hemodialysis was Identified as $\beta 2$ Microglobulin. Biochem. Biophys. Res. Commun. 1985, 129, 701-706.

32. Giles, K.; Pringle, S. D.; Worthington, K. R.; Little, D. R.; Wildgoose, J. L. Bateman, R. H. Applications of a Traveling Wave-Based Radio-FrequencyOnly Stacked Ring Ion Guide. Rapid Commun. Mass Spectrom. 2004, 18, 2401-2414.

33. Pringle, S. D. Giles, K: Wildgoose, J. L. Williams, J. P. Slade, S. E Thalassinos, K.; Bateman, R. H.; Bowers, M. T.; Scrivens, J. H. An Investigation of the Mobility Separation of Some Peptide and Protein Ions Using a New Hybrid Quadrupole/Traveling Wave IMS/oa-ToF Instrument. Int. J. Mass Spectrom. 2007, 261, 1-12.

34. Shelimov, K. B.; Clemmer, D. E.; Hudgins, R. R.; Jarrold, M. F. Protein Structure In Vacuo: The Gas Phase Conformations of BPTI and Cytochrome c. J. Am. Chem. Soc. 1997, 119, 2240-2248.

35. Valentine, S. J.; Counterman, A. E.; Clemmer, D. E. ConformerDependent Proton-Transfer Reactions of Ubiquitin Ions. J. Am. Soc. Mass Spectrom. 1997, 8, 954-961.

36. Smith, D. P.; Knapman, T. W.; Campuzano, I.; Malham, R. W.; Berryman, J. T.; Radford, S. E.; Ashcroft, A. E. Deciphering Drift Time Measurements from Traveling Wave Ion Mobility Spectrometry-Mass Spectrometry Studies. Eur. J. Mass Spectrom. 2009, 15, 113-130.

37. Ruotolo, B. T.; Benesch, J. L.; Sandercock, A. M.; Hyung, S. J.; Robinson, C. V. Ion Mobility-Mass Spectrometry Analysis of Large Protein Complexes. Nat. Protoc. 2008, 3, 1139-1152.

38. Scarff, C. A.; Thalassinos, K.; Hilton, G. R.; Scrivens, J. H. Traveling Wave Ion Mobility Mass Spectrometry Studies of Protein Structure: Biological Significance and Comparison with X-Ray Crystallography and Nuclear Magnetic Resonance Spectroscopy Measurements. Rapid Commun. Mass Spectrom. 2008, 22, 3297-3304.

39. Clemmer, D. E. http://www.indiana.edu/ clemmer/. 
40. Uetrecht, C.; Versluis, C.; Watts, N. R.; Wingfield, P. T.; Steven, A. C.; Heck, A. J. Stability and Shape of Hepatitis B Virus Capsids In Vacuo. Angew. Chem. Int. Ed. Eng. 2008, 47, 6247-6251.

41. Sobott, F.; Benesch, J. L.; Vierling, E.; Robinson, C. V. Subunit Exchange of Multimeric Protein Complexes Real-Time Monitoring of Subunit Exchange Between Small Heat Shock Proteins by Using Electrospray Mass Spectrometry. J. Biol. Chem. 2002, 277, 38921-38929.

42. Chevreux, G.; Potier, N.; Van Dorsselaer, A.; Bahoul, A.; Houdusse, A.; Wells, A.; Sweeney, H. L. Electrospray Ionization Mass Spectrometry Studies of Noncovalent Myosin VI Complexes Reveal a New Specific Calmodulin Binding Site. J. Am. Soc. Mass Spectrom. 2005, 16, 1367-1376.

43. Zal, F.; Chausson, F.; Leize, E.; Van Dorsselaer, A.; Lallier, F. H.; Green, B. N. Quadrupole Time-of-Flight Mass Spectrometry of the Native Hemocyanin of the Deep-Sea Crab Bythograea thermydron. Biomacromolecules 2002, 3, 229-231.

44. Bernstein, S. L.; Dupuis, N. F.; Lazo, N. D.; Wyttenbach, T.; Condron, M. M.; Bitan, G.; Teplow, D. B.; Shea, J. E.; Ruotolo, B. T.; Robinson, C. V.; Bowers, M. T. Amyloid- $\beta$ Protein Oligomerization and the Importance of Tetramers and Dodecamers in the Etiology of Alzheimer's Disease. Nat. Chem. 2009, 1, 326-331.

45. Smith, D. P.; Radford, S. E.; Ashcroft, A. E. Elongated Oligomers in $\beta$-2-Microglobulin Amyloid Assembly Revealed by Ion Mobility Spectrometry-Mass Spectrometry. 2010, Proc. Natl. Acad. Sci. USA, in press.

46. Wyytenbach, T.; von Helden, G.; Batka, J. J.; Carlat, D.; Bowers, M. T. Effect of the Long-Range Potential on Ion Mobility Measurements. J. Am. Soc. Mass Spectrom. 1997, 8, 275-282.

47. Mesleh, M. F.; Hunter, J. M.; Shvartsburg, A. A.; Schatz, G. C.; Jarrold, M. F. Structural Information from Ion Mobility Measurements: Effects of the Long Range Potential. J. Phys. Chem. 1996, 100, 16082-16086.

48. Shvartsburg, A. A.; Jarrold, M. F. An Exact Hard Spheres Scattering Model for the Mobilities of Polyatomic Ions. Chem. Phys. Lett. 1996, 261, $86-91$

49. Knapman, T. W.; Aggeli, A.; Ashcroft, A. E. Critical Concentrations of $\beta$-Sheet Peptide Self-Assembly Quantified Directly by Nanoelectrospray Ionization Mass Spectrometry. Rapid Commun. Mass Spectrom. 2008, 22, 1611-1614.

50. Williams, J. P.; Lough, J. A.; Campuzano, I.; Richardson, K.; Sadler, P. J. Use of Ion Mobility Mass Spectrometry and a Collision Cross-Section Algorithm to Study an Organometallic Ruthenium Anticancer Complex and Its Adducts with a DNA Oligonucleotide. Rapid Commun. Mass Spectrom. 2009, 22, 3563-3569.

51. Brockwell, D. J.; Radford, S. E. Intermediates: Ubiquitous Species on Folding Energy Landscapes? Curr. Opin. Struct. Biol. 2007, 17, 30-37.

52. Kodali, R.; Wetzel, R. Polymorphism in the Intermediates and Products of Amyloid Assembly. Curr. Opin. Struct. Biol. 2007, 17, 48-57.

53. Wetzel, R. Kinetics and Thermodynamics of Amyloid Fibril Assembly. Acc. Chem. Res. 2006, 39, 671-679.

54. Bellesia, G.; Shea, J. E. Diversity of Kinetic Pathways in Amyloid Fibril Formation. J. Chem. Phys. 2009, 131, 111101.

55. Nettleton, E. J.; Tito, P.; Sunde, M.; Bouchard, M.; Dobson, C. M.; Robinson, C. V. Characterization of the Oligomeric States of Insulin in Self-Assembly and Amyloid Fibril Formation by Mass Spectrometry. Biophys. J. 2000, 79, 1053-1065.

56. Larson, J. L.; Ko, E.; Miranker, A. D. Direct Measurement of Islet Amyloid Polypeptide Fibrillogenesis by Mass Spectrometry. Prot. Sci. 2000, 9, 427-431.

57. Smith, A. M.; Jahn, T. R.; Ashcroft, A. E.; Radford, S. E. Direct Observation of Oligomeric Species Formed in the Early Stages of Amyloid Fibril Formation Using Electrospray Ionization-Mass Spectrometry. J. Mol. Biol. 2006, 364, 9-19.

58. Merlini, G.; Bellotti, V. Molecular Mechanisms of Amyloidosis. New. Engl. J. Med. 2003, 349, 583-596.

59. Jablonowska, A.; Bakun, M.; Kupniewska-Kozak, A.; Dadlez, M. Alzheimer's Disease Amyloid $\beta$ [Abeta $(\mathrm{A} \beta)$ ] Peptide Fragment 10-30 Forms a Spectrum of Metastable Oligomers with Marked Preference for $\mathrm{N}$ to $\mathrm{N}$ and $\mathrm{C}$ to C Monomer Termini Proximity. J. Mol. Biol. 2004, 344, 1037-1049.

60. Bernstein, S. L.; Wyttenbach, T.; Baumketner, A.; Shea, J. E.; Bitan, G.; Teplow, D. B.; Bowers, M. T. Amyloid $\beta$-Protein: Monomer Structure and Early Aggregation States of A $\beta 42$ and Its Pro19 Alloform. J. Am. Chem. Soc. 2005, 127, 2075-2084.

61. Clemmer, D. E.; Valentine, S. J. Protein Oligomers Frozen in Time. Nat. Chem. 2009, 1, 257-258.
62. Murray, M. M.; Bernstein, S. L.; Nyugen, V.; Confron, M. M.; Teplow, D. B.; Bowers, M. T. Amyloid $\beta$ Protein: [Abeta40 (A $\beta 42)]$ rsqb] Inhibits Oligomerization. J. Am. Chem. Soc. 2009, 131, 6316-6317.

63. Baskakov, I. V.; Legname, G.; Baldwin, M. A.; Prusiner, S. B.; Cohen, F. E. Pathway Complexity of Prion Protein Assembly into Amyloid. J. Biol. Chem. 2002, 24, 21140-21148.

64. Grabenauer, M.; Wu, C.; Soto, P.; Shea, J. E.; Bowers, M. T. Oligomers of the Prion Protein Fragment 106-126 are Likely Assembled from $\beta$-Hairpins in Solution, and Methionine Oxidation Inhibits Assembly Without Altering the Peptide's Monomeric Conformation. J. Am. Chem. Soc. 2010, 132, 532-539.

65. Kheterpal, I.; Wetzel, R. Hydrogen/Deuterium Exchange Mass Spectrometry-a Window into Amyloid Structure. Acc. Chem. Res. 2006, $39,584-593$.

66. Hodkinson, J. P.; Jahn, T. R.; Radford, S. E.; Ashcroft, A. E. Comparing the HDX-ESI-MS Behavior of the Bound and Unbound MHC-1 Light Chain Highlights Conformational Differences. J. Am. Soc. Mass Spectrom. 2008, 20, 278-286.

67. Yao, Z. P.; Tito, P.; Robinson, C. V. Site-Specific Hydrogen Exchange of Proteins: Insights into the Structures of Amyloidogenic Intermediates. Methods Enzymol. 2005, 402, 389-402.

68. Kheterpal, I.; Chen, M.; Cook, K. D.; Wetzel, R. Structural Differences in [Abeta $(\mathrm{A} \beta)$ ] Amyloid Protofibrils and Fibrils Mapped by Hydrogen Exchange-Mass Spectrometry with On-Line Proteolytic Fragmentation. J. Mol. Biol. 2006, 361, 785-795.

69. Nazabal, A.; Dos Reis, S.; Bonneu, M.; Saupe, S. J.; Schmitter, J. M. Conformational Transition Occurring Upon Amyloid Aggregation of the HET-s Prion Protein of Podospora anserina Analyzed by Hydrogen/ Deuterium Exchange and Mass Spectrometry. Biochemistry 2003, 42, 8852-8861.

70. Nazabal, A.; Maddelein, M. L.; Bonneu, M.; Saupe, S. J.; Schmitter, J. M. Probing the Structure of the Infectious Amyloid form of the PrionForming Domain of HET-s Using High Resolution Hydrogen/ Deuterium Eexchange Monitored by Mass Spectrometry. J. Biol. Chem. 2005, 14, 13220-13228.

71. Lu, X.; Wintrode, P. L.; Surewicz, W. K. $\beta$-Sheet Core of Human Prion Protein Amyloid Fibrils as Determined by Hydrogen/Deuterium Exchange. Proc. Natl. Acad. Sci. U.S.A. 2007, 104, 1510-1515.

72. Tito, P.; Nettleton, E. J.; Robinson, C. V. Dissecting the Hydrogen Exchange Properties of Insulin Under Amyloid Fibril Forming Conditions: A Site-Specific Investigation by Mass Spectrometry. J. Mol. Biol. 2000, 303, 267-278.

73. Carulla, N.; Caddy, G. L.; Hall, D. R.; Zurdo, J.; Gairdi, M.; Feliz, M.; Giralt, E.; Robinson, C. V.; Dobson, C. M. Molecular Recycling Within Amyloid Fibrils. Nature 2005, 436, 554-558.

74. Guijarro, J. I.; Sunde, M.; Jones, J. A.; Campbell, I. D.; Dobson, C. M. Amyloid Fibril Formation by an SH3 Domain. Proc. Natl. Acad. Sci. U.S.A. 1998, 95, 4224-4228.

75. Monti, M.; Amoresano, A.; Giorgetti, S.; Bellotti, V.; Pucci, P. Limited Proteolysis in the Investigation of $\beta 2$-Microglobulin Amyloidogenic and Fibrillar States. Biochim. Biophys. Acta 2005, 1753, 44-50.

76. Myers, S. L.; Thompson, N. H.; Radford, S. E.; Ashcroft, A. E. Investigating the Structural Properties of Amyloid Fibrils Formed from 2Microglobulin Using Limited Proteolysis and Electrospray Ionization Mass Spectrometry. Rapid Commun. Mass Spectrom. 2006, 20, 1628-1636.

77. Monti, M.; Principe, S.; Giorgetti, S.; Mangione, P.; Merlini, G.; Clark, A. Bellotti, V.; Amoresano, A.; Pucci, P. Topical Investigation of Amyloid Fibrils Obtained from $\beta 2-M i c r o g l o b u l i n$. Prot. Sci. 2002, 11, 2362-2369.

78. Rand, K. D.; Zejl, M.; Jensen, O. N.; Jorgensen, T. J. D. Protein Hydrogen Exchange Measured at Single-Residue Resolution by Electron Transfer Dissociation Mass Spectrometry. Anal. Chem. 2009, 81, 5577-5584.

79. Findeis, M. A. Approaches to Discovery and Characterization of Inhibitors of Amyloid $\beta$-Peptide Polymerization. Biochim. Biophys. Acta 2000, 1502, 76-84.

80. Li, J.; Zhu, M.; Rajamani, S.; Uversky, V. N.; Fink, A. L. Rifampicin Inhibits $\alpha$-Synuclein Fibrillation and Disaggregates Fibrils. Chem. Biol. 2004, 11, 1513-1521.

81. Cheng, X.; van Breemen, R. B. Mass Spectrometry-Based Screening for Inhibitors of $\beta$-Amyloid Protein Aggregation. Anal. Chem. 2005, 77, 7012-7015.

82. Chan, P. H.; Pardon, E.; Menzer, L.; De Genst, E.; Kumita, J. R. Christodoulou, J.; Saerens, D.; Brans, A.; Bouillenne, F. Archer, D. B Robinson, C. V.; Muyldermans, S.; Matagne, A.; Redfield, C.; Wyns, L.; Dobson, C. M.; Dumoulin, M. Engineering a Camelid Antibody Fragment that Binds to the Active Site of Human Lysozyme and Inhibits Its Conversion into Amyloid Fibrils. Biochemistry 2008, 47, 11041-11054. 